\title{
Conversion from active surveillance to active treatment for prostate cancer: A qualitative analysis
}

\author{
Meredith Wallace Kazer ${ }^{1}$, Donald E. Bailey J ${ }^{2}$, Kendra Grimes ${ }^{3}$, J ohn Colberg ${ }^{4}$, William Kevin \\ Kelly
}

1. School of Nursing, Fairfield University, United States. 2. School of Nursing, Duke University, United States. 3. Hebrew Senior Life, United States. 4. Department of Surgery, School of Medicine, Yale University, United States. 5. Medical Oncology and Urology, Thomas Jefferson University, United States

Correspondence: Meredith Wallace Kazer. Address: School of Nursing, Fairfield University. Telephone: 203-254-4000 X2719. E-mail: mkazer@fairfield.edu

Received: November 7, 2011

Accepted: December 2, $2011 \quad$ Published: May 1, 2012

DOI : $10.5430 /$ jnep.v2n2p80

URL: http://dx.doi.org/10.5430/jnep.v2n2p80

\section{Abstract}

Active surveillance (AS) has been an option for prostate cancer management for many years. However, only about $10 \%$ of clinically appropriate prostate cancer patients undergo AS. The reasons for the low selection and adherence to AS are unknown. The purpose of this qualitative study was to examine the reasons why men convert from AS to active treatment. Known converters from AS to active treatment were identified through the PI's prior study with this population and participating urologists. A purposive sample of six participants was interviewed using a structured interview instrument developed by the researcher to examine reasons why participants converted from AS to active treatment. Participant recruitment was based out of a Urology Clinic in New Haven, Connecticut, USA. Four themes emerged from the analysis of research transcripts: 1) A Dangerous Wait, 2) Clinical Surveillance, 3) Need for Information \& Support, 4) The Aftermath. The results of the study will inform health care providers regarding the education and psychosocial support needs of men undergoing AS for prostate cancer.

\section{Key words}

Prostate cancer, Active surveillance, Uncertainty, Nursing education and support

\section{I ntroduction}

Prostate cancer is the most commonly diagnosed cancer in U. S. men ${ }^{[1]}$ and $85 \%$ of all prostate cancers are diagnosed in men older than 65 years ${ }^{[2]}$. With the widespread adoption of PSA screening, 58\% of men older than 50 years of age were screened in 2003, this is an increase of $17 \%$ from $2000^{[1]}$. A high proportion of prostate cancer diagnoses are now made when tumors are non-palpable and localized to the prostate gland. Many of these early stage cancers are slow-growing, do not progress, are not a threat to mortality, and do not lead to early death. Men with these types of tumors may undergo a short or long course of Active Surveillance (AS) for management of their prostate tumors. 
Active surveillance (AS) for prostate cancer is defined as initial monitoring followed by active treatment if the prostate tumor progresses to the extent that quality or quantity of life is affected. AS has been an option for prostate cancer management for many years, especially for older men with low grade and stage disease and low, stable prostate specific antigen (PSA) values. However, it is estimated that only about $10 \%$ of clinically appropriate prostate cancer patients undergo AS ${ }^{[3]}$. Many men who are clinically appropriate for AS undergo more active treatment options, despite high costs and adverse effects, and little documentation of treatment effectiveness. Other men initially select AS, but then undergo treatment in absence of progressing disease. However, all available therapeutic modalities for localized prostate cancer (radical prostatectomy, external beam radiotherapy, brachytherapy, and cryotherapy) entail significant risk of adverse effects such as sexual dysfunction, urethral strictures, urinary incontinence, and bowel problems ${ }^{[2]}$. For men with early-stage, low risk prostate cancer, AS provides an alternative to active treatment. That is, men make the decision to actively monitor their disease with the knowledge that treatment remains an option ${ }^{[4]}$.

The benefits of AS lie in the avoidance of expensive treatments that have well-documented adverse effects. Treatment adverse events are prevalent among men after these procedures. In a study of 326 Swedish men aged 48 to 74 undergoing AS $(n=187)$ or radical prostatectomy $(n=189)$, Steineck et al ${ }^{[5]}$ reported a higher frequency of both erectile dysfunction (80\% versus $45 \%)$ and urinary leakage (49\% versus $21 \%$ ) in radical prostatectomy patients compared to the men undergoing AS. Dall'Era et al ${ }^{[6]}$ report that delaying treatment through AS allows men to benefit from further advances in available treatments.

Men who undergo AS live every day with the knowledge that they have cancer and the associated uncertainty about whether the cancer will grow, spread, and result in death. In a qualitative study by Wallace and Storms ${ }^{[7]}$ on the needs of men with prostate cancer, one focus group participant stated, "the word cancer is followed closely in my mind with death." Moreover, research has found that even when men are asymptomatic or experience only occasional signs that the cancer is present; their concern about the state of their cancer is intense ${ }^{[8]}$. Except for bi-annual PSA readings, which are inherently wrought with uncertainty, men undergoing AS have no clinical markers to indicate disease progression, and thus may incorrectly attribute physical changes associated with aging to disease progression ${ }^{[8]}$. Other men, who experience no physical discomfort, find it hard to believe that the cancer exists. Wallace and Storms ${ }^{[7]}$ also found in their focus group study, that men with prostate cancer were highly influenced by family members in making their treatment decision.

There are many reasons why men who are clinically appropriate for AS convert to active treatment. In some cases, the prostate cancer progresses to the extent where AS is no longer an option and active treatment in the form of radical prostatectomy and radiotherapy are necessary. Men without education and support may also experience emotional distress such as depression and choose to undergo unnecessary treatment ${ }^{[8]}$. Disease uncertainty associated with prostate cancer is a significant event and may be so great that men opt to convert to treatment in the form of radical prostatectomy or radiotherapy to simply "get rid of it" than to continue to live with the possibility of the cancer growing and leading to death. The specific aim of this study is to use a qualitative approach to examine the reasons why men undergoing AS convert to active treatment. The results of the study will inform health care providers regarding the education and psychosocial support needs of men undergoing AS for prostate cancer.

\section{Methods}

This study was approved by the Yale University School of Nursing/Human Subjects Research Review Committee and Yale New Haven Hospital/Nursing Research Committee. A purposive, sample of six men previously undergoing AS who converted to active treatment were recruited to participate in this study. This sampling method was chosen to ensure that participants had purposely undergone a defined period of AS before converting to active treatment and could thus fully enlighten the researcher in the area of investigation. Eligible patients were identified from the clinic schedule by participating urologists in the eastern United States. Participants received mailed invitations to participate the study, Published by Sciedu Press 
including a cover letter and study flyer. The letter to participants requested them to call the PI if interested in participating. When the potential participant contacted the investigator, the purpose and description of the study were discussed and the participant was asked if he would be interested in scheduling an interview. Participants were given the opportunity to either decline participation or be scheduled to participate. If the participant agreed to the interview, the research assistant contacted them to set up a mutually convenient time and location for the interview.

The inclusion criteria was a period of at least six months in AS followed by active treatment for prostate cancer, aged 60 and older; fluency in English and ability to give informed consent. The period of six months in AS was important to truly represent that men in the study were in AS and not simply awaiting a time when treatment was conveniently implemented. As the average life span for adult males in the U.S. is approximately 80 years old and data reveals that AS "is feasible and appears safe in the 10- to 15-year time frame" ${ }^{[9]}$ the minimum age of 60 was considered appropriate for AS and participation in this study. The research question for this study was, why do men undergoing AS for prostate cancer convert to active treatment for prostate cancer? Participants were asked to describe their experiences with diagnosis and the decision to both undergo AS and active treatment, formal and informal sources of support and information, problems they experienced in AS and active treatment, and facilitators and barriers to the decision making process (see Table 1). A demographic questionnaire was used to gather data on age, ethnicity and marital status in order to describe the sample.

Table 1. Interview Questions

1. Describe your experience of being diagnosed with prostate cancer.

2. How did you go about initially choosing your active surveillance?

Did you turn to anyone for advice or support? Is yes, who?

3. During your period on active surveillance, did you have a relative or friend who helped you: with any emotional difficulties? Obtain information about prostate cancer or your treatment? If no, what was it like going through this experience without any help?

4. What was most difficult about your period on active surveillance? Were you worried that your cancer was growing? Did you have difficulty explaining active surveillance to your family or friends? What factor/s influenced your decision to convert to active surveillance the most?

5. How did you go about selecting your active treatment for prostate cancer?

Did you turn to anyone for advice or support? Is yes, who?

6. Are you experiencing any problems in your life now due to having had prostate cancer treatment? Who or what services help you with these problems

a. Physically?

b. Sexually?

c. Emotionally?

d. Socially?

e. In everyday activities?

7. If you had your whole prostate cancer experience to do over again, would you do anything differently?

8. Anything else you want to share?

Interviews were conducted at the time and place agreed upon by both the research assistant and research participant. After introductions were completed, informed consent and other necessary research authorizations and questionnaires were 
completed by the research assistant. Following this, the researcher turned on the audio recorder and began the interview. Permissible probes were used as necessary.

\section{Results}

A total of six participants met the study criteria, agreed to participate and completed the interviews. Participants' ages ranged from 60 to 78 with a mean age of 70 . All participants were Caucasian, five were married and one participant was single. Two participants had radiotherapy, two had radical prostatectomies and two had hormone treatments. A fundamental qualitative methodology was used to analyze the results of the interviews. This methodology "entails the presentation of the facts of the case in everyday language" ${ }^{[10]}$. In this manner, data collection and analysis occurs concurrently beginning with the first subject. Analysis was focused on identifying and categorizing each participant's comments. Interviews were audio taped and transcribed. Audiotapes were transcribed using a word processing program and proofed by the investigators. The investigator read the transcription, while listening to the audiotapes, coding the data in the margin of the transcript as appropriate and organizing the responses into possible categories to determine an initial consensus regarding the essential themes of the interviews by noting the frequencies of similar messages. Additional data were analyzed, and categories clarified and changed. All analytic strategies were documented.

Four themes emerged from the analysis of research transcripts: 1) A Dangerous Wait; 2) Clinical Surveillance; 3) Need for Information and Support; 4) The Aftermath.

\subsection{A dangerous wait}

Participants commonly experienced a period of apprehension or anxiety during AS which may be described as "dangerous waiting”. This experience appears similar to that of uncertainty which frequently accompanies the experience of cancer and other chronic illnesses. Uncertainty plays a significant role in explaining various outcomes in the chronically ill. However, little is known about the uncertainty that accompanies AS. This period or apprehension or uncertainty was described by one 79 year old white, single, high-school educated participant who had been treated with radiation. He reported:

"It's a dangerous wait because by the time they get it tested and so on and so on, it'd probably be too late".

Two study participants explained the contradiction between the AS management option amidst the pressure of other treatment options. They described this with the following quotes:

A 65 year old, white, married, masters-educated participant who had received hormone therapy treatment for prostate cancer explained that:

"Everybody said, I should do something, I should do something”.

A 65 year old, white, married, masters-educated participant who had undergone a radical prostatectomy shared a discussion he had with a friend:

“He couldn't understand why I would even risk my life in this way”.

\subsection{Clinical surveillance}

As seen in table 1, participants were asked to begin the interview by telling their story about prostate cancer diagnosis. Each participant began their story with a discussion of the Prostate Specific Antigen (PSA) level that led to their diagnosis and proceeded through detailing the clinical surveillance of their prostate cancer diagnosis, multiple biopsies, period of time in AS and eventual treatment. 
A 79 year old white, single, high-school educated participant who had been treated with radiation reported:

"I had a PSA...maybe it's under 4, then a year later it was 5... They took a biopsy and it was cancer".

One 68 year old white, married college-educated participant who had undergone a radical prostatectomy discussed that:

"Your numbers are starting to jump around a little bit 7 to 8 1/2".

A 65 year old, white, married, masters-educated participant who had undergone a radical prostatectomy shared that:

"I had a PSA level that was up and down".

A 72 year old, white, married, masters-educated participant who had received hormone therapy treatment for prostate cancer informed investigators that:

"I should go and get it done, because by the report, by the biopsy, it's getting worse".

\subsection{Need for information \& support}

During the same time that participants were going through the clinical course of their disease, they commenced in a period of seeking information and support. This period took on different forms for different patients, but a few commonalities were found. Specifically, all men in this study found a "prostate buddy" or someone who played an important role in their information and support seeking process. For some men this person was an established friend who also had been diagnosed with prostate cancer. For others, the prostate buddy was someone they met after diagnosis through their physician or a formal support network. This person played a key role in the participants' experience by answering questions, vetting and helping to make decisions.

Through the entire experience, participants sought support from family members or acquaintances that had experienced prostate cancer. One 68 year old white, married college-educated participant who had undergone a radical prostatectomy discussed that:

"You need an advocate with you, you need somebody that's not afraid to ask questions and raise hell".

Men looked to a variety of sources of information about prostate cancer, including written materials and their physician. However, most men commented that the internet was a common place to start the search for prostate cancer knowledge. A 79 year old white, single, high-school educated participant who had been treated with radiation reported:

"I was looking at the computer for prostate treatment".

One 68 year old white, married college-educated participant who had undergone a radical prostatectomy discussed that:

"I went on a couple of websites".

\subsection{The aftermath}

Men were asked to discuss their treatment decision and life after diagnosis with prostate cancer. Of the six men in the study two had radical prostatectomies, two had radiotherapy, two eventually proceeded to hormone therapy. Overall, men were experiencing typical and well-documented psychosocial and physical outcomes of cancer treatment.

Without much prompting, men discussed the multiple concerns experienced during the aftermath of treatment. Most men in the study openly discussed issues with sexual dysfunction and all men reported concerns with treatment failure or disease recurrence. This was marked by continued PSA monitoring similar to that experienced during their AS period as well as a candid discussion of the adverse effects of treatment. One 68 year old white, married college-educated participant who had undergone a radical prostatectomy discussed that: 
"The Incontinence - minor inconveniences. They got these pads they sell...I just need one of them real thin ones".

A 79 year old white, single, high-school educated participant who had been treated with radiation reported:

"I had a little problem with sex for a little bit".

A 72 year old, white, married, masters-educated participant who had received hormone therapy treatment for prostate cancer informed investigators that:

"I had no interest in sex".

\section{Discussion}

AS offers men with early stage prostate cancer an alternative to prostate cancer treatment and the adverse events that often accompany treatment. However, little is known about the experiences of men in AS. Findings from the interviews provide insight into the kinds of concerns those men undergoing AS face, the processes they use to manage concerns, support needed during AS and possible explanations for converting from AS to active treatment.

Men in this study felt that living with prostate cancer was a dangerous thing to do. Given that men undergoing AS live every day with the knowledge that they have cancer in their bodies, it is understandable that uncertainty about the state of cancer may intensify commensurate with clinical disease progression. Among men in AS who may be asymptomatic or experience only occasional signs that the cancer is present, it is difficult to believe that cancer exists. Thus, men may incorrectly attribute physical changes associated with aging to disease progression ${ }^{[4]}$. The uncertainty associated with AS is supported by early work by Wallace ${ }^{[11]}$ who conducted a descriptive study aimed at exploring the extent to which uncertainty, anxiety, and primary appraisal of danger explained the health-related and affective QOL of men who were receiving AS for prostate cancer. In the national sample of 19 men, significant relationships were found between uncertainty, anxiety, and appraisal of danger and the affective physical function domain of QOL $(r=0.553 ; p=0.014)$. The addition of appraisal of danger explained a significant amount of the variance in QOL above uncertainty alone, underscoring the potential role of danger appraisal in overall QOL ${ }^{[11]}$. It is important that these prevalent concerns of uncertainty and danger appraisal be incorporated into psychosocial and educational interventions of men undergoing AS. Moreover, if AS is to succeed, patients must be offered appropriate interventions as they seek to manage the inevitable and constant uncertainty about living with cancer. Inability to do so may cause patients to deteriorate emotionally or to undergo unnecessary treatment ${ }^{[12]}$.

The pattern of clinical surveillance that all participants in this study reported typifies the prostate cancer experience. Prostate cancer is usually detected through a routine PSA screening test and diagnosis is confirmed by clinical data that includes patient report of urinary symptoms, digital rectal exam (DRE), transrectal ultrasound (TRUS), and positive findings from prostate biopsy ${ }^{[12]}$. Usual PSA values are between $0-4.0 \mathrm{ng} / \mathrm{mL}$; values greater than 4.0 may require additional follow-up dependent on a number of patient characteristics including, but not limited to age, ethnicity, body mass index, height, and a family history of prostate cancer, and values over $10.0 \mathrm{ng} / \mathrm{ml}$ are highly suspicious of prostate cancer. Men in this study clearly followed clinical protocols and tracked their PSA values with their providers. In the case of all participants in this study, rising PSA values led to eventual treatment for prostate cancer. However, it is important to note that there are numerous treatment options for prostate cancer and clarity about when to begin treatment and which treatment options are best for which patient is needed. Further study is also needed in order to differentiate between indolent and aggressive tumor characteristics as well as biomarkers for prostate cancer in order to better customize treatments for future prostate cancer patients. Moreover, there is significant controversy regarding whether the PSA test should be used to screen men for the presence of prostate cancer as existing the evidence does not support routine population screening with PSA and testing has been associated with psychological harm ${ }^{[13]}$. The U.S. Preventive Services 
Task force has recently recommended that men over 75 years of age forgo PSA screening due to limited benefit and increased risk for physical and psychological harm ${ }^{[14]}$.

The participants in this study consistently turned to family, friends and the internet for information related to prostate cancer and AS. The use of the internet to deliver interventions is a new approach to the delivery of care with the potential for international, asynchronous dissemination. Wantland et al ${ }^{[15]}$ report that the years 1996 to 2003 witnessed a 12 -fold increase in MEDLINE citations for "Web-based therapies". Kirsch and Lewis ${ }^{[16]}$ conducted a comprehensive review of the literature regarding the use and efficacy of internet-based health care interventions. In their review of nine published studies, the authors found that "research suggests that Web-based interventions may be an efficacious delivery system, especially for those with chronic conditions amenable to self-management and to those with various limitations to accessing healthcare”.

The aftermath of living with prostate cancer as seen among this sample, is wrought with management of the adverse effects of prostate cancer treatment. Surgical and radiotherapy treatments to the prostate gland and surrounding tissue generally result in the undesirable adverse events of erectile dysfunction (ED) and urinary incontinence. The rate of erectile dysfunction following radical prostatectomy has been reported to be greater than $80 \%{ }^{[17]}$ and $10 \%$ to $30 \%$ of patients undergoing external beam radiotherapy ${ }^{[18]}$. Current research supports the use of oral erectile agents (phosphodiesterase type 5 (PDE5) inhibitors) to manage erectile dysfunction (ED) following radical prostatectomy ${ }^{[19]}$. Urinary incontinence (UI) ranges from $5 \%$ to over $60 \%{ }^{[20]}$ among men undergoing radical prostatectomy, but is a lesser problem among men undergoing radiotherapy ${ }^{[21]}$. Hormone treatments impact libido and erectile function, as well as produce many other deleterious systemic effects, but do not result in urinary adverse effects ${ }^{[22]}$.

\section{Limitations}

This study was limited by the small number of participants, the subjective nature of the study design, and the homogeneity of the sample. However, this study population has never been the subject of prior study. Identifying the needs of men with prostate cancer is an essential step in understanding the experience of men with this disease. Thus, the information provided herein offers essential data necessary to develop information and support services for men in AS. The findings from this study may be used to enhance current support services to meet identified needs of men with prostate cancer. Future study may examine the role of health care providers in meeting the needs of men undergoing AS for prostate cancer, especially in patient teaching, and the development, implementation and evaluation services and internet interventions.

\section{Summary and conclusion}

The AS management option for prostate cancer has been available for many years, but continues to lack popularity among patients and providers. The reasons for this are unknown. This small qualitative study aimed at examining the reasons why men convert from AS to treatment revealed that most men convert to treatment because of a clinical indication. However, PSA surveillance of prostate cancer growth may not be the most sensitive indicator of disease progression. Thus, more research is needed in order to distinguish indolent from aggressive tumors and appropriate treatment options for prostate cancer. Men in AS have continually reported the need for support and information throughout the cancer trajectory and this was supported by this study. Men with prostate cancer may benefit from both one-on-one and group support networks. Educational needs may be filled by enhanced education within urology practices, or effective internet interventions as indicated by the sample in this study. Research has shown that AS results in intolerable uncertainty that impacts quality of life to the extent that it is actually preferable to undergo treatment to simply "get rid of it" than to continue to live with the 
possibility of the cancer growing and being killed. Thus, interventions for men undergoing AS must include elements of uncertainty management and management of symptoms related to aftermath of prostate cancer treatment.

\section{Sources of support}

This research was supported by a Research Grant from the Delta Mu Chapter of Sigma Theta Tau International Honor Society of Nursing.

\section{Conflict of interest}

The authors have no conflicts of interest to declare in the publication of this manuscript.

\section{References}

[1] American Cancer Society. American Cancer Society. Cancer Facts \& Figures. 2008. Atlanta: American Cancer Society.

[2] Gronberg, H. Prostate cancer epidemiology. Lancet. 2003; 361: 859-64. http://dx.doi.org/10.1016/S0140-6736(03)12713-4

[3] Cooperberg, M. R., Broering, J. M., Kantoff, P. W., \& Carroll, P. R. Contemporary trends in low risk prostate cancer: risk assessment and treatment. Journal of Urology. 2007; 178: S14-S19. PMid:17644125 http://dx.doi.org/10.1016/j.juro.2007.03.135

[4] Bailey DE,Jr, Wallace M, Mishel MH. Watching, waiting and uncertainty in prostate cancer. J Clin Nurs. 2007; 16(4):734-741. PMid:17402955 http://dx.doi.org/10.1111/j.1365-2702.2005.01545.x

[5] Steineck, G., Helgesen, F., Adolfsson, J., Dickman, P. W., Johansson, J. E., Norlen, B. J., \& Holmberg, L. Scandinavian Prostatic Cancer Group Study Number 4. Quality of life after radical prostatectomy or watchful waiting. New England Journal of Medicine .2002; 347: 790-796. PMid:12226149 http://dx.doi.org/10.1056/NEJMoa021483

[6] Dall'Era MA, Cooperberg MR, Chan JM, et al. Active surveillance for early-stage prostate cancer: review of the current literature .Cancer. 2008; 112(8):1650-1659. PMid:18306379 http://dx.doi.org/10.1002/cncr.23373

[7] Wallace, M., \& Storms, S. The needs of men with prostate cancer. Applied Nursing Research. 2007; 20(4):181-187. PMid:17996804 http://dx.doi.org/10.1016/j.apnr.2006.08.008

[8] Bailey, D. E., Mishel, M. H., Belyea, M., Stewart, J. L., Mohler, J. Uncertainty intervention for watchful waiting in prostate cancer. Cancer Nursing. 2004; 27: 339. PMid:15525860 http://dx.doi.org/10.1097/00002820-200409000-00001

[9] Klotz, L. Zhang, L. Lam, A., Nam, R., Mamedov, A. \& Loblaw, A. Clinical Results of Long-Term Follow-Up of a Large, Active Surveillance Cohort With Localized Prostate Cancer. Journal of Clinical Oncology. 2010; 28 (1): 126-131. PMid:19917860 http://dx.doi.org/10.1200/JCO.2009.24.2180

[10] Sandelowski, M. What happened to qualitative description? Research in Nursing \& Health. 2000; $23: 334-340$. http://dx.doi.org/10.1002/1098-240X(200008)23:4<334::AID-NUR9>3.0.CO;2-G

[11] Wallace, M. The quality of life of men undergoing watchful waiting for prostate cancer. Oncology Nursing Forum. 2003; 30: 303-309. PMid:12692664 http://dx.doi.org/10.1188/03.ONF.303-309

[12] Wallace, M. Bailey, D.E., \& Brion, J. Prostate Cancer Update. NP Journal. 2009; 34(10): 24-34.

[13] Ilic D, O'Connor D, Green S, Wilt T. Screening for prostate cancer. Cochrane Database Syst Rev. 2006; 3:004720.

[14] Lin K, Lipsitz R, Miller T, Janakiraman S, U.S, Preventive Services Task F. Benefits and harms of prostate-specific antigen screening for prostate cancer: an evidence update for the U.S. Preventive Services Task Force. Ann Intern Med. 2008; 149(3): 192-199.PMid:18678846

[15] U.S. Preventive Services Task Force. Screening for prostate cancer: US Preventive Services Task Force Recommendations statement. Ann Intern Med. 2008; 149:185-191. PMid:18678845

[16] Wantland, D. J., Portillo, C. J., Holzemer, W. L., Slaughter, R., \& McGhee, E. M. The effectiveness of Web-based vs. non-Webbased interventions: A meta-analysis of behavioral change outcomes. Journal of Medical Internet Research. 2004 ; 6(4), e40. PMid:15631964 http://dx.doi.org/10.2196/jmir.6.4.e40

[17] Kirsch SE. Lewis FM. Using the World Wide Web in health-related intervention research. A review of controlled trials. Computers, Informatics, Nursing. 2004 Jan-Feb; 22(1):8-18. http://dx.doi.org/10.1097/00024665-200401000-00005

[18] Siegel T, Moul JW, Spevak M, Alvord WG, Costabile RA. The development of erectile dysfunction in men treated for prostate cancer. J Urol. 2001; 165(2):430-435. PMid:11176390 http://dx.doi.org/10.1097/00005392-200102000-00019

[19] Bott SR, Birtle AJ, Taylor CJ, Kirby RS. Prostate cancer management: (1) an update on localised disease. Postgrad Med J. 2003; 79(936):575-580. PMid:14612600 http://dx.doi.org/10.1136/pmj.79.936.575

Published by Sciedu Press 
[20] Miles CL, Candy B, Jones L, Williams R, Tookman A, King M. Interventions for sexual dysfunction following treatments for cancer. Cochrane Database Syst Rev. 2007; 4:005540.

[21] Hunskaar S, Burgio K, Diokno AC, Herzog AR, Hjalmas K, Lapitan MC. Epidemiology and natural history of urinary incontinence. Incontinence: 2nd International Consultation on Incontinence. Plymouth, UK: Health Publications. 2002; 165-200.

[22] Harrington JM, Jones EG, Badger T. Body image perceptions in men with prostate cancer. Oncol Nurs Forum. 2009; 36(2): 167-172. PMid:19273405 http://dx.doi.org/10.1188/09.ONF.167-172 